Research Article

\title{
Human ADMC-Derived Adipocyte Thermogenic Capacity Is Regulated by IL-4 Receptor
}

\author{
Fernando Lizcano, Diana Vargas, Ángela Gómez, and Astrid Torrado \\ Center of Biomedical Investigation from University of La Sabana (CIBUS), Chí, Cundinamarca, Colombia \\ Correspondence should be addressed to Fernando Lizcano; fernando.lizcano@unisabana.edu.co
}

Received 7 June 2017; Accepted 24 August 2017; Published 12 October 2017

Academic Editor: Luca Vanella

Copyright (C) 2017 Fernando Lizcano et al. This is an open access article distributed under the Creative Commons Attribution License, which permits unrestricted use, distribution, and reproduction in any medium, provided the original work is properly cited.

\begin{abstract}
Type two innate immune system is anti-inflammatory and may play an important role as the means whereby "browning" is induced in subcutaneous adipocytes. It was shown that IL-4 may influence the fate of adipose cell precursors by promoting differentiation towards more thermogenic adipocytes in mice. Here, we investigated the influence of IL-4 and IL-4 receptor, a type two immune cytokine pathway, on the metabolic activity and thermogenic potential of human adipocytes differentiated from adipose-derived mesenchymal stem cells (ADMSCs) obtained from subcutaneous samples of healthy women undergoing abdominoplasty. Western blot analysis, qPCR, and biochemical analyses were performed 10 days after ADMSC differentiation into mature adipocytes was induced. IL-4 receptor was expressed in both precursor and differentiated adipocytes, and IL- 4 treatment increased phosphorylation Y641 of signal transducer and activator of transcription 6 (STAT6) in both cell types. IL-4 treatment also increased expression of thermogenic proteins PGC- $1 \alpha$, UCP-1, and CITED1. In addition, IL- 4 increased the secretion of adiponectin, leptin, and FGF21 and promoted lipolysis in differentiated adipocytes. In conclusion, IL-4 may directly modulate differentiation of human adipocytes towards a beige phenotype acting through IL-4 receptors on both adipose precursors and differentiated human adipocytes, metabolic effect that must be considered in some antiallergic drugs.
\end{abstract}

\section{Introduction}

Obesity is associated with a state of mild inflammation, which can contribute to insulin resistance and type 2 diabetes mellitus [1]. This mild inflammatory condition is characterized by changes in the macrophage population in adipose tissue $[2,3]$. An increase in proinflammatory type-1 macrophages (M1), which promotes insulin resistance, is evident in the adipose tissue of obese people, who also demonstrate reduced numbers of type-2 macrophages (M2), which are insulin sensitizing $[4,5]$.

Several studies have emphasized the role of interleukins (ILs) in the physiology of adipose cells: ILs increase lipolysis and modify body fat distribution and production of extracellular matrix by adipose tissue [6-8]. However, it was suggested that the interaction between adipose cells and the immune system is more complex than previously thought [9]. Some studies have shown that the activation of group 2 innate lymphoid cells (ILC2s) may modify the function of adipose tissue and systemic glucose homeostasis. It was observed that after IL-33 stimulation, eosinophils and ILC2s produce IL-4 and IL-13 [10-13], which are ILs that may promote the differentiation of adipose precursors into "brown like" adipocytes [14]. Additionally, IL-33 can influence the production of met-enkephalin by ILC2s, which can induce the process of "browning" in differentiated adipose cells [10]. Additionally, M2 macrophages that are activated by IL-4 can be mobilized in the subcutaneous adipose tissue, secreting catecholamines that activate brown adipocytes and induce the differentiation of beige adipocytes [14, 15]. We previously observed an elevation of thermogenic gene expression induced by cold that was increased by IL- 4 in human adipocytes [16].

Despite the observed effect of IL- 4 on the differentiation of beige adipocytes from precursor cells, the effects of IL-4 on differentiated adipocytes and its influence on the process of browning have yet to be investigated. Previous studies showed reduced IL-4 receptor (IL-4R) expression in 


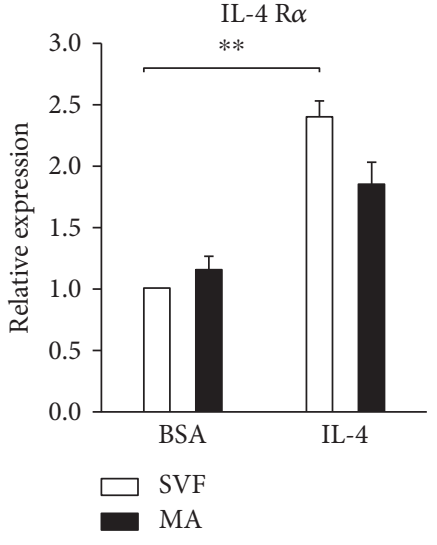

(a)

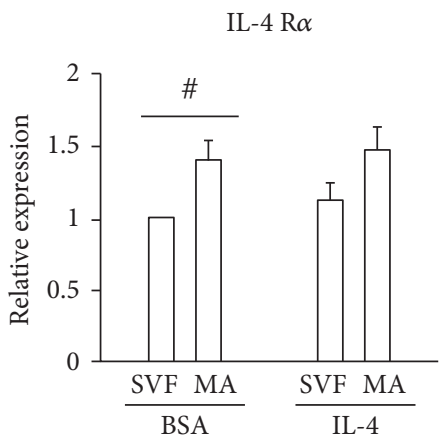

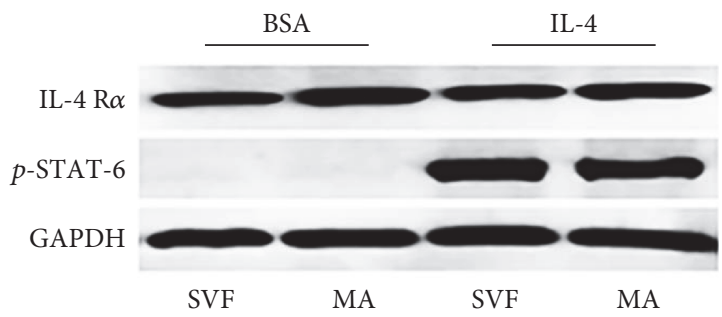

(b)

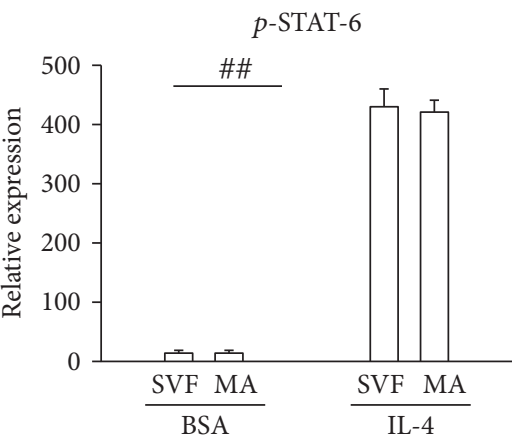

(c)

FIgURE 1: Expression of IL-4R $\alpha$ and pSTAT-6 in precursor and mature adipocytes. Precursor cells (SVF) were obtained from human subcutaneous adipose tissue, induced to proliferate, and then differentiated into mature adipocytes (MA), when they were treated with BSA alone (vehicle) or IL-4. (a) IL-4R mRNA expression was quantified by qPCR using GAPDH as a reference gene. Data represent mean \pm SD. ${ }^{* *} p<0.01$. (b) IL-4R and phosphorylated STAT-6 (pSTAT-6) protein were measured using Western blot. (c) Relative band intensity was determined by densitometry. Statistical analyses were performed using ANOVA. Data represent mean \pm SD. ${ }^{\#} p<0.05$ between precursors (SVF) and differentiated adipocytes (MA) after $6 \mathrm{~h}$ of BSA or IL-4 treatment. ${ }^{\# \#} p<0.001$ between precursors (SVF) and differentiated adipocytes (MA) before and after $6 \mathrm{~h}$ of IL-4 treatment. Data were normalized to GAPDH as reference gene/protein.

differentiated adipose cells. However, these studies were primarily performed in 3 T3-L1 cells and mouse adipose tissue $[11,17]$. Therefore, the functional roles of IL-4 and its receptor remain to be determined in human differentiated adipocytes. On the other hand, it recently was observed that the adaptive thermogenesis is not commonly associated to a chronic treatment with IL-4 in mice. Fischer et al. reported opposite results with respect to the previous assessment of relevant amounts of catecholamine synthesis by IL- 4 alternatively activated macrophages, which involved a role of IL-4 in adaptive thermogenesis and brown/beige adipocyte metabolism [18]. The regular signaling pathway of IL-4 is initiated by binding of ligand to type I receptors (IL- $4 \mathrm{R} \alpha / \gamma$-chain) and type II receptors (IL-4R $\alpha / \mathrm{IL}-13 \mathrm{R} \alpha 1$ ) [19]. The binding of IL-4 to IL-4R induces receptor dimerization, which activates an intracellular signaling pathway [20] that involves the activation of the transcription factor signal transducer and activator of transcription 6 (STAT6) and insulin receptor substrate $2[21,22]$.

To add to the limited knowledge regarding the influence of IL- 4 on the browning process in humans, we studied the phenotypic characteristics of differentiated adipocytes from healthy women undergoing lipectomy. We observed that IL-4 treatment induced phenotypic characteristics typical of beige adipocytes in differentiated white adipocytes. Additionally, we found that both precursor cells and differentiated adipocytes express IL-4R, and IL- 4 induces an increase in phosphorylation of STAT6. This implies that IL-4 may have a direct effect on differentiated adipocytes through IL-4R, resulting in "browning."

\section{Materials and Methods}

Subcutaneous fat samples were obtained from eight healthy women aged 20-40 years, who were undergoing abdominoplasty. They had body mass indexes (BMIs) of $23-25 \mathrm{~kg} / \mathrm{m}^{2}$ and had received no drug treatment during the 3 months prior to sampling and did not show any signs of disease. In addition, their lipid profiles and glucose levels were within the normal range. They received detailed information regarding the purpose of the study and gave informed consent. The project was approved by the ethics committee of the University of La Sabana. 


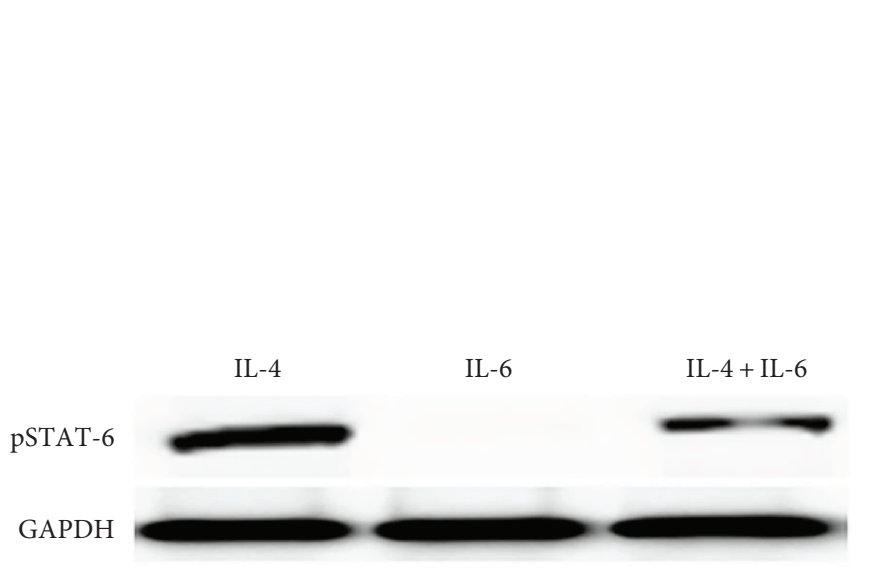

(a)

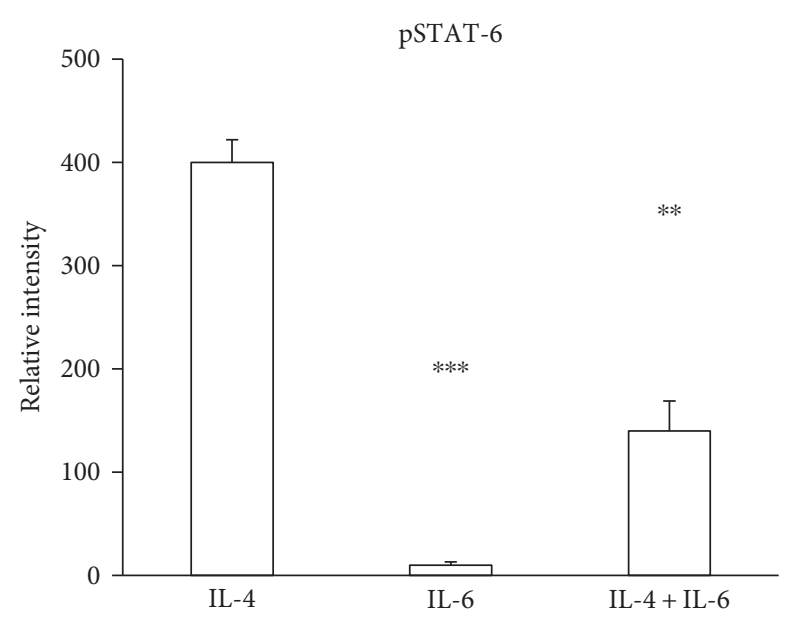

(b)

FIgURE 2: pSTAT-6 is induced by IL-4 treatment of mature adipocytes. Precursor cells were obtained, proliferated, and induced to differentiate into mature adipocytes, followed by treatment with BSA alone (vehicle), IL-4, $10 \mathrm{ng} / \mathrm{mL}$; IL-6, $100 \mathrm{ng} / \mathrm{mL}$, or IL-4, $10 \mathrm{ng} / \mathrm{mL}$ and IL-6, $100 \mathrm{ng} / \mathrm{mL}$. (a) Phosphorylated STAT-6 (pSTAT-6) protein was measured using Western blot. (b) Phosphorylated STAT-6 (pSTAT-6) protein expression was measured by Western blotting. Relative band intensity was determined by densitometry. Statistical analyses were performed using ANOVA. Data represent mean \pm SD. ${ }^{* *} p<0.01$ between mature adipocytes treated with IL-4 and IL-4 + IL-6. ${ }^{* * *} p<0.001$ between mature adipocytes (MA) treated with IL-4 or IL-6.

2.1. Cell Culture and Differentiation. Precursor cells were isolated from $30 \mathrm{~g}$ abdominal subcutaneous adipose tissue obtained during surgery. Adipose samples were washed with phosphate-buffered saline (PBS), and all visible fibrous material and blood vessels were removed. Subsequently, samples were digested with $250 \mathrm{U} / \mathrm{mL}$ type I collagenase, $20 \mathrm{mg} / \mathrm{mL}$ bovine serum albumin (BSA), and $60 \mu \mathrm{g} / \mathrm{mL}$ gentamicin in PBS for $90 \mathrm{~min}$ at $37^{\circ} \mathrm{C}$. After digestion, the samples were centrifuged at $200 \times \mathrm{g}$ for $10 \mathrm{~min}$, and the pellet was resuspended in an erythrocyte lysis solution containing $154 \mathrm{mM}$ ammonium chloride, $5.7 \mathrm{mM}$ monobasic potassium phosphate, and $0.1 \mathrm{mM}$ EDTA $\mathrm{pH} 7.3$ for $10 \mathrm{~min}$. This mixture was filtered through a nylon mesh (pore size: $150 \mu \mathrm{m}$ ), followed by centrifugation at $200 \times \mathrm{g}$ for $10 \mathrm{~min}$. The cell pellet was then resuspended in proliferation media containing Dulbecco's Modified Eagle Medium (DMEM)/F12, 10\% v/v fetal bovine serum, and $50 \mu \mathrm{g} / \mathrm{mL}$ gentamicin and seeded at a density of 10,000 cells $/ \mathrm{cm}^{2}$.

After $24 \mathrm{~h}$, the cells were washed and induced to proliferate by addition of PM4 media (DMEM/F12 containing 2.5\% fetal bovine serum, $1 \mathrm{ng} / \mathrm{mL}$ basic fibroblast growth factor, $10 \mathrm{ng} / \mathrm{mL}$ epidermal growth factor, and $8.7 \mu \mathrm{M}$ insulin). When cells had reached $100 \%$ confluency, human precursor cells were differentiated into adipocytes by addition of DMEM/F12 medium containing $66 \mathrm{nmol} \mathrm{L}^{-1}$ insulin, $1 \mathrm{nmol}$ $\mathrm{L}^{-1}$ triiodo-L-thyronine, $10 \mu \mathrm{g} / \mathrm{mL}$ transferrin, $0.5 \mathrm{mmol} \mathrm{L}^{-1}$ isobutyl-methylxanthine, $100 \mathrm{nmol} \quad \mathrm{L}^{-1}$ dexamethasone, and $1 \mu \mathrm{mol} \mathrm{L} \mathrm{L}^{-1}$ rosiglitazone (a peroxisome proliferatoractivated receptor $\gamma$ (PPAR $\gamma)$ agonist) for $72 \mathrm{~h}$. Subsequently, the medium was replaced with preadipocyte basal medium containing the same concentrations of insulin, triiodo-L-thyronine, and transferrin for 10 days, with changes every 3 days. The effect of IL- 4 on mature adipocytes was evaluated by treatment with $10 \mathrm{ng} / \mathrm{mL}$ of IL- 4
(PeproTech, Rocky Hill, NJ, USA) in $0.1 \% w / v$ BSA for $6 \mathrm{~h}$. As a negative control, mature adipocytes were treated with 100 ng/mL IL6 (Cell Signaling Technology, Danvers, MA, USA) in $0.1 \% w / v$ BSA for $6 \mathrm{~h}$.

2.2. Adipocyte Cold Induction. In previous studies, performed by our group, primary human adipose cells were evaluated and observed that a reduction on temperature from $37^{\circ} \mathrm{C}$ to $30^{\circ} \mathrm{C}$ during a period may resemble a cold induction with an increase of thermogenic proteins, UCP- 1 and PGC- $1 \alpha$.

Adipocyte precursor cells were induced to differentiate by culturing in 6-well plates for 10 days at $37^{\circ} \mathrm{C}$ in basal differentiation medium. Cells were washed with PBS, basal medium was added, and then cells were incubated at $30^{\circ} \mathrm{C}$ in $5 \% \mathrm{CO}_{2}$ and in the presence or absence of $10 \mathrm{ng} / \mathrm{mL}$ IL-4 for $6 \mathrm{~h}$. Cells were then lysed for analysis for protein expression. Control cells were cultured under the same conditions at $37^{\circ} \mathrm{C}[16]$.

2.3. $q P C R$. Total RNA was isolated from both human adipocyte precursor cells and differentiated adipocytes. RNA extraction was performed using a High Pure RNA Isolation Kit (Roche Diagnostics, Mannheim, Germany) in accordance with the manufacturer's instructions. Then, $500 \mathrm{ng}$ RNA was used to generate cDNA using a Transcriptor First Strand cDNA Synthesis Kit (Roche Diagnostics, Mannheim, Germany). The following primers were used for gene expression detection, following the recommended protocol for use with the FastStart Essential DNA Green Master Mix (Roche Diagnostics, Mannheim, Germany): IL-4R Fw: $5^{\prime}$ GTGCTATGTCAGCATCACCAAGA 3', Rev: $5^{\prime}$ CCC CTGAGCATCCTGGATTAT $3^{\prime}$ and uncoupling protein 1 (UCP-1) Fw: 5' GTGTGCCCAACTGTGCAATG 3', Rev: 5' CCAGGATCCAAGTCGCAAGA 3'. Quantitative analyses 


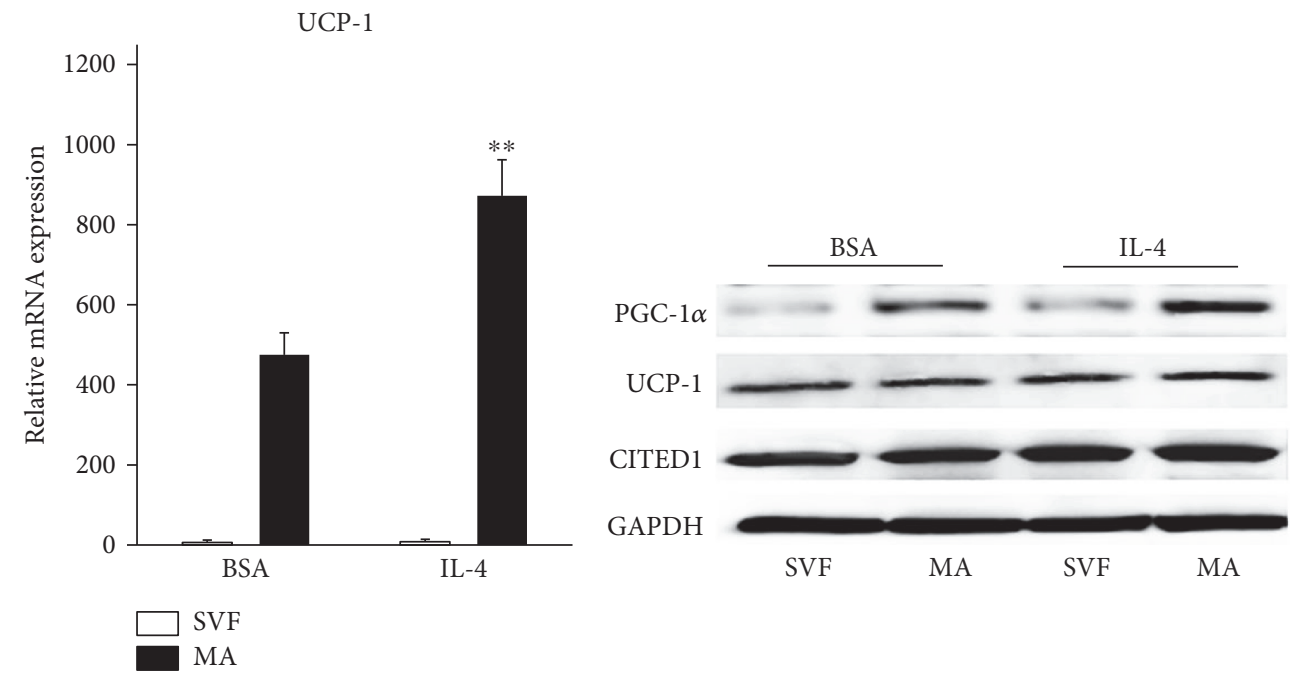

(a)

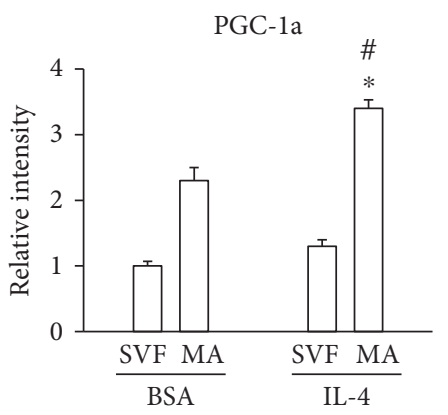

UCP1

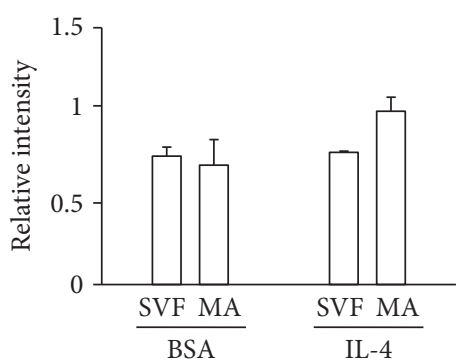

(b)

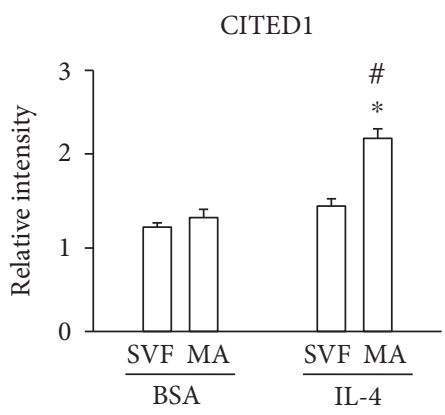

(c)

FIGURE 3: Effect of IL-4 on the expression of thermogenic genes. Precursor cells were obtained, proliferated, and induced to differentiate into mature adipocytes and then treated with BSA alone (vehicle) or IL-4. (a) UCP-1 mRNA expression was measured by qPCR. (b) Protein expression of PGC-1 $\alpha$, UCP-1, and CITED1 was measured by Western blotting. (c) Relative band intensities for each were determined by densitometry. Statistical analyses were performed using ANOVA. Data represent mean \pm SD and were normalized to GAPDH as reference gene/protein. ${ }^{*} p<0.05$ between precursors (SVF) and mature adipocytes (MA) after $6 \mathrm{~h}$ of IL- 4 treatment, ${ }^{* *} p<0.01$ relative RNA expression between mature adipocytes treated with vehicle or IL-4, and ${ }^{\#} p<0.05$ between mature adipocytes treated with IL-4 or vehicle.

of mRNA expression levels were normalized to expression of glyceraldehyde-3-phosphate dehydrogenase (GAPDH; primer sequences: Fw 5' ACCCACTCCTCCACCTTTGAC $3^{\prime}$ and Rev 5' TGTTGCTGTAGCCAAATTCGTT $3^{\prime}$ using the $\Delta \Delta \mathrm{Ct}$ method.

2.4. Western Blot Analysis. Adipocytes differentiated from subcutaneous adipose tissue were lysed in radioimmunoprecipitation assay (RIPA) buffer (Abcam, Cambridge, MA, USA; ab156034) containing $1 \mu$ g protease inhibitor cocktail (Roche Diagnostics, Mannheim, Germany). Total protein content of each lysate was then quantified using the Bradford method, and the lysates were diluted to a final working concentration of $50 \mu \mathrm{g} / \mathrm{mL}$ protein. Lysates were denatured at $95^{\circ} \mathrm{C}$ and subjected to polyacrylamide gel electrophoresis. Products were then electrotransferred to polyvinylidene difluoride membranes pretreated with $100 \%$ methanol for $2 \mathrm{~min}$. Blocking was performed in PBS-T (1x PBS containing $0.1 \%$ Tween 20 ) containing $5 \% w / v$ skimmed milk powder.

Membranes were then incubated with antibodies targeting proteins involved in thermogenesis: rabbit anti-PGC-1 $\alpha$
(1:1000, Abcam ab54481), rabbit anti-UCP-1 (1:1000, Abcam ab155117), rabbit anti-IL-4R (1:1000, Abcam ab131058), rabbit anti-STAT6 phosphorylated (phospho Y641) (1:1000, Abcam ab54461), and mouse anti-cAMP response element-binding protein/p300-interacting transactivator 1 (CITED1; Abcam ab87978). Secondary antibodies against rabbit IgG, conjugated to horseradish peroxidase (IgG-HRP) for PGC-1 $\alpha$, IL-4R, and pSTAT6 (1:5000) and UCP1 $(1: 3000)$, were used. Mouse IgG-HRP was used as the secondary antibody following anti-CITED1 application $(1: 2000)$.

Expression levels of adipokines were measured using rabbit anti-fatty acid-binding protein 4 (FABP4; $1: 3000$, Abcam $92,501)$, anti-adiponectin $(1: 3000$, Abcam ab92501), fibroblast growth factor 21 (FGF21; 1:1000, Abcam ab171941), and leptin (Abcam ab16227), with rabbit IgG-HRP (1:5000) being used as a secondary antibody. Detection was performed by chemiluminescence using the Luminata Crescendo Kit (Millipore, Billerica, MA, USA). Images were captured and analyzed using myECL Imager, a blot and gel documentation instrument (Catalog number 62236, Thermo Scientific, 


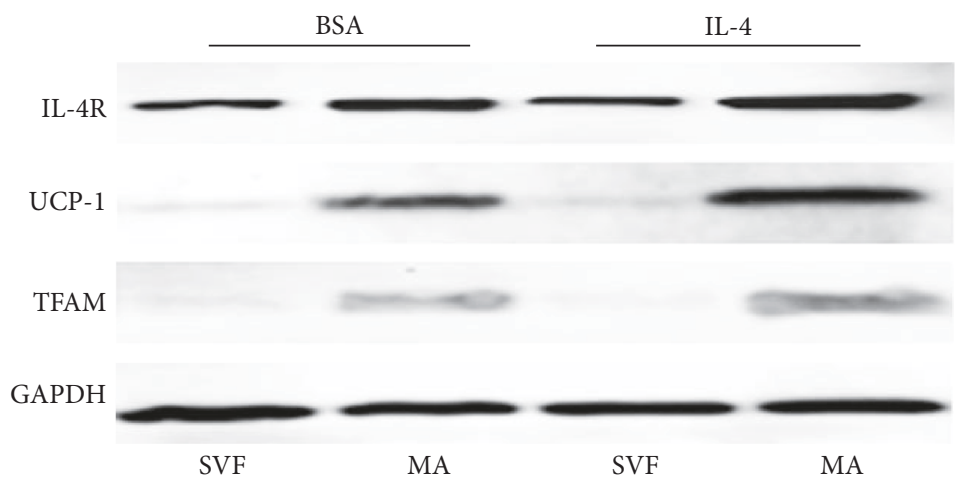

(a)
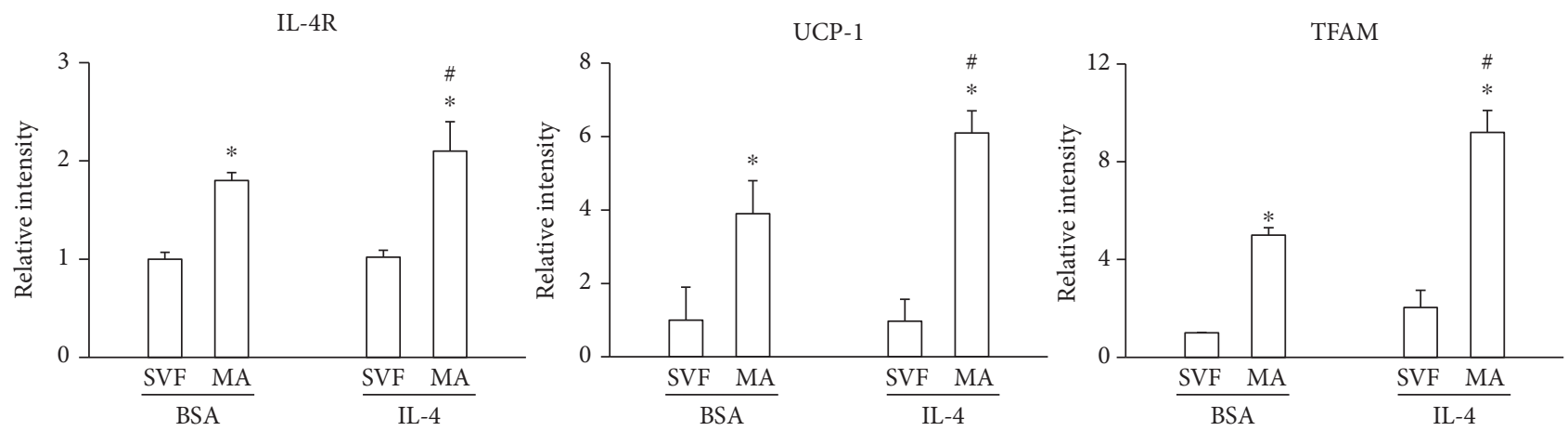

(b)

FIGURE 4: IL-4 treatment increases expression of mitochondrial proteins. Precursors (SVF) were obtained and differentiated as described in Materials and Methods. Cells were treated with BSA alone (vehicle) or IL- 4 and incubated at $31^{\circ} \mathrm{C}$ for $6 \mathrm{~h}$, then lysed. (a) IL-4R, UCP-1, and TFAM protein expression were measured by Western blotting. (b) Relative band intensity of each was determined by densitometry. Statistical analyses were performed using ANOVA. Data represent mean \pm SD and were normalized to expression of the reference protein GAPDH. ${ }^{*} p<0.05$ between precursors (SVF) and mature adipocytes (MA). ${ }^{*} p<0.05$ between mature adipocytes (MA) treated with IL-4 or vehicle.

Waltham, MA, USA). Quantitative densitometric analysis was performed using My Image Analysis software for three independent experiments.

2.5. Lipolysis Assay. Precursors and differentiated adipocytes were washed and incubated in assay buffer in the presence of BSA as a carrier or were treated with $10 \mathrm{ng} / \mathrm{mL}$ IL-4. As a positive control, cells were treated with $1 \mu \mathrm{M}$ isoproterenol. Glycerol content of the supernatant was measured using a Cultured Human Adipocyte Lipolysis Assay Kit (Zen-Bio, Research Triangle Park, NC, USA) following the manufacturer's instructions, with spectrophotometric detection at $540 \mathrm{~nm}$.

2.6. Statistical Analysis. Data were evaluated using one-way ANOVA, data represent means \pm SD of three independent experiments from the samples of 8 patients which proteins were analyzed by Western blot. The differences between means were performed through Student's $t$-test. Differences were considered statistically significant when $p<0.05$.

\section{Results}

3.1. IL-4 Activates the IL-4R Signaling Pathway in Precursors and Differentiated Adipocytes. This study builds on previous experiments in which mouse adipocyte precursors treated with IL-4 demonstrated a beige phenotype, following activation of the IL-4R/STAT- 6 pathway. By contrast, differentiated adipocytes were thought to undergo "browning" because of the activation of a different pathway, because they do not express IL-4R $[11,14]$. Adipose-derived mesenchymal stem cells (ADMSCs) from human subcutaneous fat were induced to differentiate for 10 days. The expression of IL- $4 \mathrm{R}$ was measured in adipocyte precursors (stromal vascular fraction (SVF)) and adipocytes were induced to differentiate using a differentiation cocktail (mature adipocytes), before and after treatment with IL-4.

As shown in Figure 1(a), both precursor and mature adipocytes expressed IL-4R mRNA and IL-4 treatment significantly increased the expression of IL- $4 \mathrm{R}$ in both types of cells. Previous studies showed that IL-4 can induce browning in precursor adipocytes through IL-4R [11]. However, IL-4R was shown not to be expressed by adipocytes derived from murine precursor cells. We aimed to evaluate the role of IL- $4 \mathrm{R}$ in differentiated adipocytes from human subcutaneous adipose tissue; therefore, protein levels of IL-4R and pSTAT-6 were determined. IL-4R protein was expressed in both precursors and differentiated adipocytes, and pSTAT- 6 was increased 


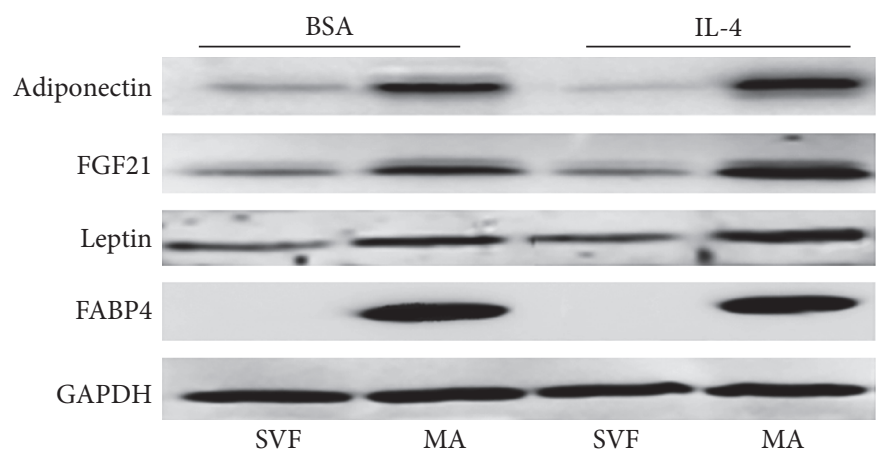

(a)
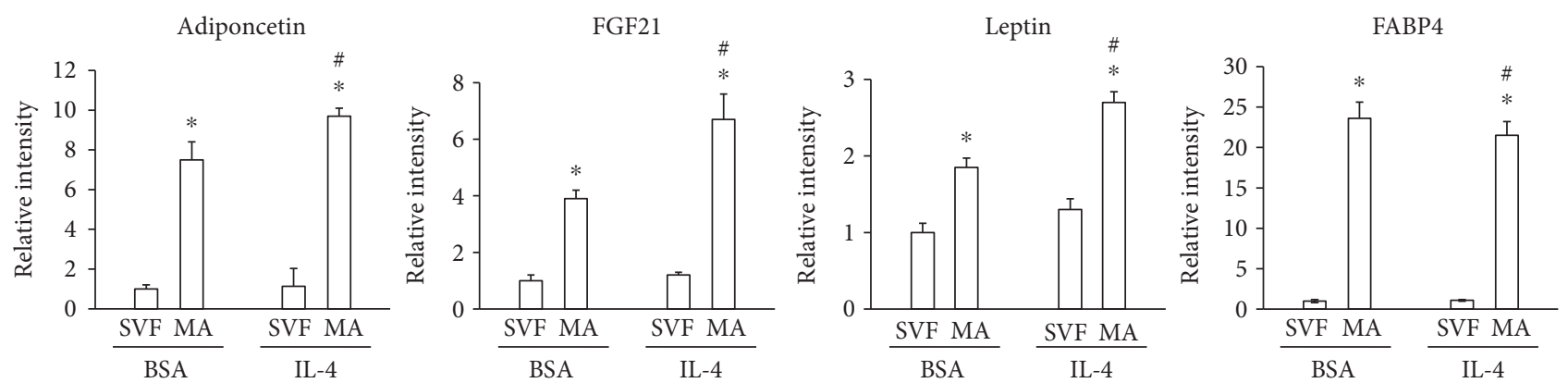

(b)

FIGURE 5: Effect of IL-4 on adipocytokine expression by adipocytes. Precursor cells were obtained, proliferated, and induced to differentiate into mature adipocytes, then treated with BSA alone (vehicle) or IL-4. (a) Adiponectin, FGF21, leptin, and FAB4 protein expression were measured by Western blotting. (b) Relative band intensity of each was determined by densitometry. Statistical analyses were performed using ANOVA. Data represent mean \pm SD and were normalized to the reference protein GAPDH. ${ }^{*} p<0.05$ between precursors (SVF) and mature adipocytes (MA). ${ }^{*} p<0.05$ between mature adipocytes (MA) treated with IL-4 and vehicle.

by the administration of IL-4 to both cell types (Figures $1(\mathrm{~b})$ and $1(\mathrm{c}))$.

3.2. pSTAT-6 Is Induced by IL-4 in Mature Adipocytes. To establish whether the IL-4 signaling pathway is activated following IL-4 treatment of mature adipocytes, we assayed pSTAT-6. Mature adipocytes were treated with IL-4, IL-6, or IL- 4 and IL-6. As expected, pSTAT- 6 was detected in adipocytes treated with IL-4, while IL- 6 treatment did not induce pSTAT-6 (Figures 2(a) and 2(b)).

3.3. Effect of IL-4 on Thermogenic Protein Expression. To determine whether treatment with IL-4 in mature adipocytes could induce browning, precursor cells and mature adipocytes were stimulated with $10 \mathrm{ng} / \mathrm{mL} \mathrm{IL-} 4$ for $6 \mathrm{~h}$. UCP-1 mRNA was shown to be induced in the presence of IL-4 (Figure 3(a)), and, as shown in Figures 3(b) and 3(c), protein expression levels of PGC- $1 \alpha$ and CITED1 were increased by 2.3 - and nearly 2 -fold, respectively.

3.4. IL-4 Increases Mitochondrial Protein Activity. To investigate the effect of IL-4 on mitochondrial activity, mature adipocytes were treated with IL- 4 and incubated at $31^{\circ} \mathrm{C}$ for $6 \mathrm{~h}$. The incubation to $31^{\circ} \mathrm{C}$ resembles a reduction of temperature and remark the effect on UCP1. Cell lysates were then prepared, and protein expression of UCP1 and TFAM was measured by Western blotting. IL-4 enhanced the hypothermia-induced increases in mitochondrial protein expression (Figures 4(a) and 4(b)), implying a browning effect of IL-4 in mature adipocytes.

3.5. Effect of IL-4 on the Expression of Adipokines in Differentiated Adipocytes. A major challenge in evaluating the induction of browning is the characterization of its effect on the production of adipokines. To assess whether IL-4 treatment of mature adipocytes was capable of inducing proteins involved in lipid and glucose metabolism, the expression of adipokines that influence relevant pathways was assessed. IL-4 increased the expression of adiponectin, leptin, and FGF21 in mature adipocytes compared with cells treated with BSA alone (Figures 5(a) and 5(b)). Expression of FABP4 confirmed that the cells were mature adipocytes.

3.6. IL-4 Enhances Hypothermia-Induced Activation of Thermogenesis. Adiponectin is an adipokine known for its protective effect against cardiometabolic diseases and diabetes mellitus. Exposure to cold in humans and mice induces the production of adiponectin, in part through activation of the IL-4 pathway. To evaluate whether the IL- 4 treatment might increase hypothermia-induced adiponectin expression, adipocytes were exposed to low temperature $\left(31^{\circ} \mathrm{C}\right)$ and treated with $10 \mathrm{ng} / \mathrm{mL}$ IL-4. This resulted in increased expression of adiponectin and PGC- $1 \alpha$ in adipocytes, 


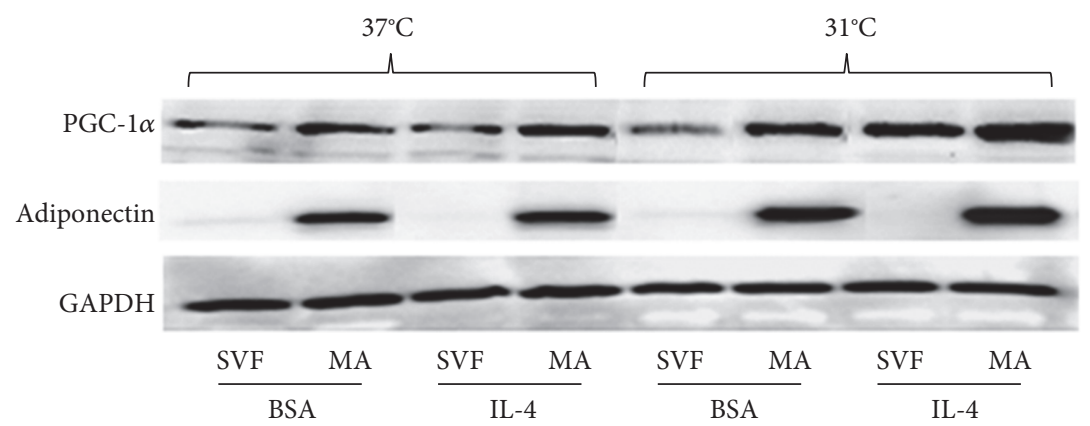

(a)
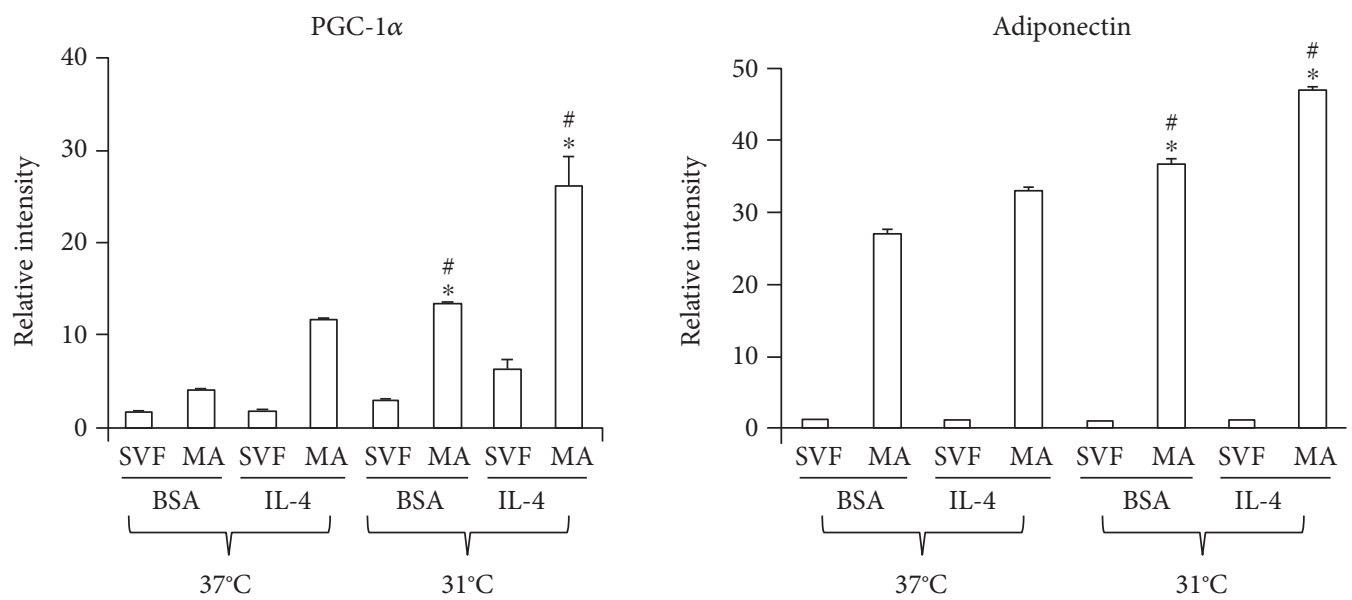

(b)

Figure 6: Induction of proteins associated with thermogenesis by cold and IL-4. Precursors were obtained and differentiated as described in Materials and Methods. Cells were treated with BSA alone (vehicle) or IL- 4 and incubated at $37^{\circ} \mathrm{C}$ or $31^{\circ} \mathrm{C}$ for $6 \mathrm{~h}$. (a) PGC- $1 \alpha$ and adiponectin protein expression were determined by Western blotting of cell lysates. (b). Relative band intensity of each was determined by densitometry. Statistical analyses were performed using ANOVA. Data represent mean \pm SD and were normalized to the reference protein GAPDH. ${ }^{*} p<0.05$ between the differentiated adipocytes exposed to $37^{\circ} \mathrm{C}$ and those exposed to $31^{\circ} \mathrm{C}$. ${ }^{*} p<0.05$ between differentiated adipocytes exposed to $37^{\circ} \mathrm{C}$ and those exposed to $31^{\circ} \mathrm{C}$, in the presence of IL-4.

compared to cells exposed to the same conditions of temperature but treated with vehicle (Figures 6(a) and 6(b)).

3.7. Lipolytic Effect of IL-4. Our results imply that IL-4 may modulate the phenotype of mature adipocytes by promoting browning and inducing expression of adipokines involved in lipid and carbohydrate metabolism. Therefore, we were prompted to evaluate whether IL- 4 could modulate lipid mobilization in the mature adipocyte. To this end, we examined the effect of IL-4 on lipolysis in mature adipocytes and showed that IL-4 treatment for $6 \mathrm{~h}$ increased lipolysis (Figure 7).

\section{Discussion}

This work highlights the role of IL-4 receptor (IL-4R) in promoting browning of mature human adipocytes. These adipocyte cells were differentiated from ADMSCs that had been obtained during abdominoplasty in women with normal BMI. IL-4 treatment of the differentiated cells resulted in increased expression levels of pSTAT-6, PGC- $1 \alpha$, UCP-1, and CITED1. These findings suggest that IL- 4 could be

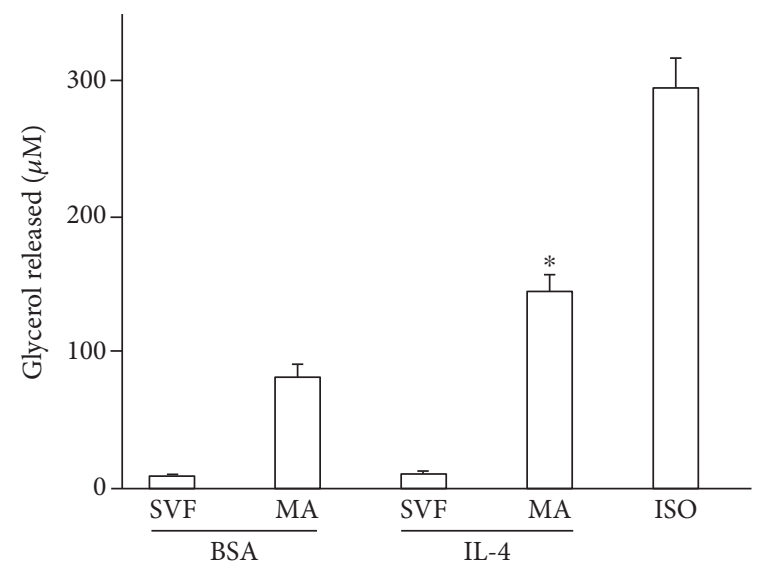

Figure 7: Lipolytic effect of IL-4. Precursor cells were obtained and differentiated as described in Materials and Methods, then treated with BSA alone (vehicle), IL-4, or isoproterenol for 6 or $24 \mathrm{~h}$. The culture media was recovered, and the glycerol concentration was determined. Statistical analyses were performed using ANOVA. Data represent mean $\pm \mathrm{SD}$. ${ }^{*} p<0.05$ between mature adipocytes treated with vehicle or IL-4. 


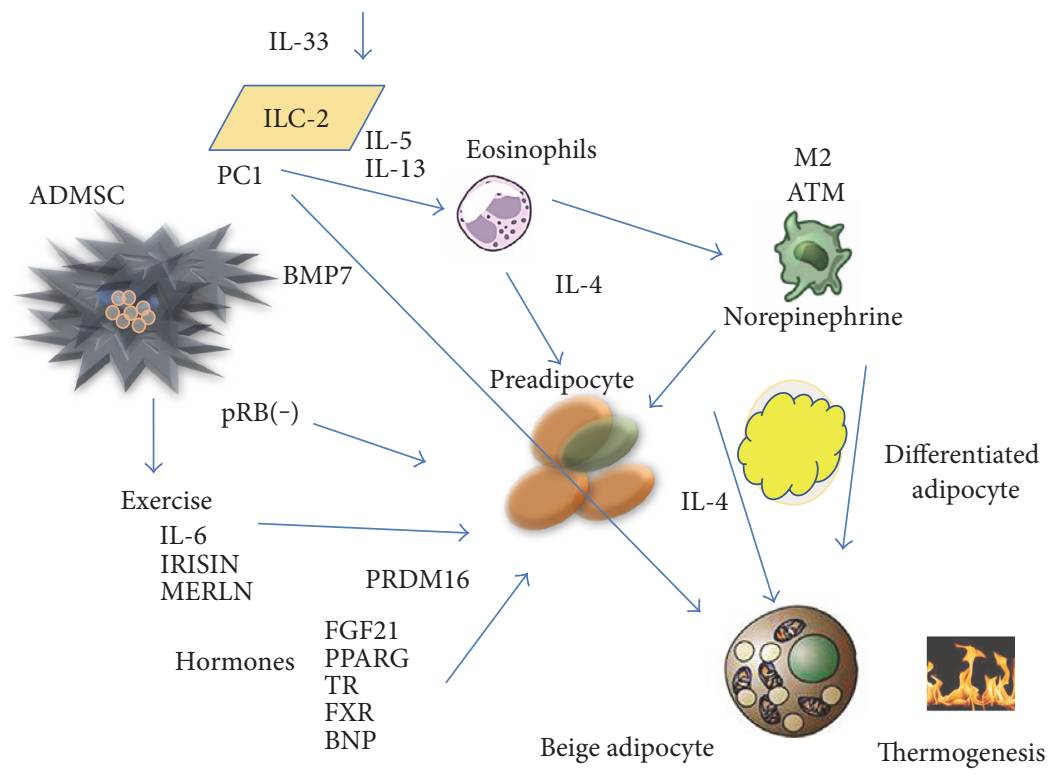

FIGURE 8: Determining factors in differentiation of beige adipose cell in humans. The adipose mesenchymal cells can be influenced by the retinoblastoma protein $(\mathrm{pRb})$ and take the decision to differentiate into fat cells when $\mathrm{pRb}$ is blocked. BMP7 triggers production of mesenchymal adipose cells to brown adipose cells. Both exercise and some hormones can increase the capacity of adipose stem cells to differentiate into beige adipocytes. Recently, it has been observed that cells of the innate immune system type 2 can secrete interleukins stimulating the production of IL- 4 by eosinophils and norepinephrine production through macrophage type 2 . IL-33 can activate the differentiation of beige adipocytes directly. ADMSC: adipose mesenchymal stem cell; PC1: Prohormone Convertase 1; ILC-2: group 2 innate lymphoid cells; BMP7: bone morphogenic protein 7; M2 ATM: adipose tissue macrophage 2; FGF21: fibroblastic growth factor 21; PPAR $\gamma$ : peroxisome proliferator-activated receptor gamma; TR: thyroid receptors; FXR: farnesoid X receptor; BNP: brain natriuretic factor.

exerting a direct browning effect on adipocytes by binding to IL- $4 \mathrm{R} \alpha$ receptors. Although some studies have considered the role of IL- 4 and its receptor in adipocyte browning, these were mainly of adipocyte precursors [11,23], which preferentially differentiated into beige adipocytes in the presence of IL-4.

Previously, Qiu and colleagues suggested that IL-4 produced by eosinophils stimulated catecholamine production by M2 macrophages, which induced a change in adipocyte phenotype towards that of beige adipocytes [14]. Other studies showed that ILC2s do not require IL-4 to induce browning; instead, they may induce this effect by increased met-enkephalin production after stimulation with IL-33 [10]. Recently, it was observed that during the winter season the browning process increases, an event mediated by IL- 4 and mast cells [24]. The present work showed that IL-4R in humans and pSTAT-6 are expressed in differentiated adipocytes and that IL- 4 treatment increases expression of PGC- $1 \alpha$, UCP-1, and CITED1.

In addition, IL-4 treatment increases expression levels of adipokines in adipocytes, including those of adiponectin and FGF21 [25, 26], in which the effects are metabolically beneficial. This finding is relevant because, in addition to increasing thermogenesis in adult adipocytes, IL- 4 may have an additional favorable effect on carbohydrate metabolism. Both adiponectin and FGF21 may improve glucose uptake by peripheral tissues and insulin sensitivity [27, 28].

The effect of IL- 4 on the expression of proteins implicated in thermogenesis in mature adipocytes was accompanied by an increase in lipolysis shown by increased glycerol release into the medium (Figure 5). A further interesting observation was the increased expression levels of UCP-1, TFAM, PGC- $1 \alpha$, and adiponectin when the cells were subjected to low temperature, as shown in Figures 4 and 6 . The response of the cells to cold stress and the mechanism for acclimatization to environmental cold are mediated in part by type 2 immune cells (ILC2s, eosinophils, and activated macrophages) that produce interleukins (IL-4, IL-13, and IL-33) [11]. It was previously demonstrated that cold can enable the induction of a beige phenotype in cultured adipocytes, which is mediated by IL-4, among other factors [16, 29]. Our observations in the present work showed that further increase in the thermogenic proteins observed with temperature reduction exhibits additional mechanisms than the only effect of IL-4 and IL-4R. Other factors that may contribute to the outcome of temperature reduction on the increase in thermogenic proteins in the adipocytes can be the activation of adrenergic receptors and methionine-enkephalin [30-32]. However, more research is required to address this question. Given that previous studies that observed reductions in IL-4R expression were performed in mouse and rat adipocyte cell lines, we believe that this study is novel in demonstrating the involvement of IL-4 and its receptor in the browning of mature adipocytes derived from human ADMSCs. It will be important to analyze the metabolic behavior of patients who are treated with specific blockers of IL-4R in therapies for atopic diseases. These data imply that targeting IL-4R to encourage increased energy expenditure by adipocytes may 
be of use in the treatment of obesity. A schematic role of ILs in the browning process is shown in Figure 8.

\section{Disclosure}

The founding sponsors had no role in the design of the study; in the collection, analyses, or interpretation of data; in the writing of the manuscript; and in the decision to publish the results.

\section{Conflicts of Interest}

The authors declare no conflict of interest.

\section{Authors' Contributions}

Fernando Lizcano and Diana Vargas conceived and designed the experiments. Diana Vargas and Fernando Lizcano analyzed the data. Ángela Gómez and Astrid Torrado contributed reagents/materials/analysis tools. Fernando Lizcano and Diana Vargas wrote the paper.

\section{Acknowledgments}

This research was supported by Colciencias 519/2011 and La Sabana University MED-181-2014. Thanks to DIN (Research and the School of Medicine from La Universidad de La Sabana) for logistical and economic support. Special thanks to Dra. Leonor Botero and Dr. Juan Duque for their collaboration.

\section{References}

[1] S. Klein, N. F. Sheard, X. Pi-Sunyer et al., "Weight management through lifestyle modification for the prevention and management of type 2 diabetes: rationale and strategies: a statement of the American Diabetes Association, the North American Association for the Study of Obesity, and the American Society for Clinical Nutrition," Diabetes Care, vol. 27, no. 8, pp. 20672073, 2004.

[2] C. E. Juge-Aubry, E. Somm, R. Chicheportiche et al., "Regulatory effects of interleukin (IL)-1, interferon- $\beta$, and IL- 4 on the production of IL-1 receptor antagonist by human adipose tissue," The Journal of Clinical Endocrinology and Metabolism, vol. 89, no. 6, pp. 2652-2658, 2004.

[3] Z. S. Nagy, Z. Czimmerer, A. Szanto, and L. Nagy, "Proinflammatory cytokines negatively regulate $\operatorname{PPAR} \gamma$ mediated gene expression in both human and murine macrophages via multiple mechanisms," Immunobiology, vol. 218, no. 11, pp. 1336-1344, 2013.

[4] C. N. Lumeng, J. L. Bodzin, and A. R. Saltiel, "Obesity induces a phenotypic switch in adipose tissue macrophage polarization," The Journal of Clinical Investigation, vol. 117, no. 1, pp. 175-184, 2007.

[5] A. S. Molgat, A. Gagnon, C. Foster, and A. Sorisky, "The activation state of macrophages alters their ability to suppress preadipocyte apoptosis," The Journal of Endocrinology, vol. 214, no. 1, pp. 21-29, 2012.

[6] A.S. Morisset, C. Huot, D. Legare, and A. Tchernof, "Circulating IL-6 concentrations and abdominal adipocyte isoproterenolstimulated lipolysis in women," Obesity, vol. 16, no. 7, pp. 1487-1492, 2008.
[7] P. Morigny, M. Houssier, E. Mouisel, and D. Langin, "Adipocyte lipolysis and insulin resistance," Biochimie, vol. 125, pp. 259-266, 2016.

[8] G. S. Hotamisligil, "Inflammation and metabolic disorders," Nature, vol. 444, no. 7121, pp. 860-867, 2006.

[9] H. Kwon and J. E. Pessin, "Adipokines mediate inflammation and insulin resistance," Frontiers Endocrinology, vol. 4, p. 71, 2013.

[10] J. R. Brestoff, B. S. Kim, S. A. Saenz et al., "Group 2 innate lymphoid cells promote beiging of white adipose tissue and limit obesity," Nature, vol. 519, no. 7542, pp. 242-246, 2015.

[11] M. W. Lee, J. I. Odegaard, L. Mukundan et al., "Activated type 2 innate lymphoid cells regulate beige fat biogenesis," Cell, vol. 160, no. 1-2, pp. 74-87, 2015.

[12] D. Wu, A. B. Molofsky, H. E. Liang et al., "Eosinophils sustain adipose alternatively activated macrophages associated with glucose homeostasis," Science, vol. 332, no. 6026, pp. 243247, 2011.

[13] P. S. Liu, Y. W. Lin, F. H. Burton, and L. N. Wei, "Injecting engineered anti-inflammatory macrophages therapeutically induces white adipose tissue browning and improves dietinduced insulin resistance," Adipocytes, vol. 4, no. 2, pp. 123128, 2015.

[14] Y. Qiu, K. D. Nguyen, J. I. Odegaard et al., "Eosinophils and type 2 cytokine signaling in macrophages orchestrate development of functional beige fat," Cell, vol. 157, no. 6, pp. 1292-1308, 2014.

[15] K. D. Nguyen, Y. Qiu, X. Cui et al., "Alternatively activated macrophages produce catecholamines to sustain adaptive thermogenesis," Nature, vol. 480, no. 7375, pp. 104-108, 2011.

[16] D. Vargas, W. Rosales, and F. Lizcano, "Modifications of human subcutaneous ADMSC after PPAR $\gamma$ activation and cold exposition," Stem Cells International, vol. 2015, Article ID 196348, 8 pages, 2015.

[17] C. H. Tsao, M. Y. Shiau, P. H. Chuang, Y. H. Chang, and J. Hwang, "Interleukin-4 regulates lipid metabolism by inhibiting adipogenesis and promoting lipolysis," Journal of Lipid Research, vol. 55, no. 3, pp. 385-397, 2014.

[18] K. Fischer, H. H. Ruiz, K. Jhun et al., "Alternatively activated macrophages do not synthesize catecholamines or contribute to adipose tissue adaptive thermogenesis," Nature Medicine, vol. 23, no. 5, pp. 623-630, 2017.

[19] S. J. Van Dyken and R. M. Locksley, "Interleukin-4- and interleukin-13-mediated alternatively activated macrophages: roles in homeostasis and disease," Annual Review of Immunology, vol. 31, pp. 317-343, 2013.

[20] A. E. Kelly-Welch, E. M. Hanson, M. R. Boothby, and A. D. Keegan, "Interleukin-4 and interleukin-13 signaling connections maps,” Science, vol. 300, no. 5625, pp. 1527-1528, 2003.

[21] S. Goenka and M. H. Kaplan, "Transcriptional regulation by STAT6," Immunologic Research, vol. 50, no. 1, pp. 87-96, 2011.

[22] E. Maier, A. Duschl, and J. Horejs-Hoeck, "STAT6-dependent and -independent mechanisms in Th2 polarization," European Journal of Immunology, vol. 42, no. 11, pp. 2827-2833, 2012.

[23] S. D. Lee and P. Tontonoz, "Eosinophils in fat: pink is the new brown," Cell, vol. 157, no. 6, pp. 1249-1250, 2014.

[24] J. Park, M. Kim, K. Sun, Y. A. An, X. Gu, and P. E. Scherer, "VEGF-A-Expressing Adipose Tissue Shows Rapid Beiging and Enhanced Survival After Transplantation and Confers 
IL-4-Independent Metabolic Improvements," Diabetes, vol. 66, no. 6, pp. 1479-1490, 2017.

[25] F. M. Fisher, S. Kleiner, N. Douris et al., "FGF21 regulates PGC- $1 \alpha$ and browning of white adipose tissues in adaptive thermogenesis," Genes \& Development, vol. 26, no. 3, pp. 271-281, 2012.

[26] A. Kharitonenkov, T. L. Shiyanova, A. Koester et al., "FGF-21 as a novel metabolic regulator," The Journal of Clinical Investigation, vol. 115, no. 6, pp. 1627-1635, 2005.

[27] M. Claussnitzer, S. N. Dankel, K. H. Kim et al., "FTO obesity variant circuitry and adipocyte browning in humans," The New England Journal of Medicine, vol. 373, no. 10, pp. 895907, 2015.

[28] X. Hui, P. Gu, J. Zhang et al., “Adiponectin enhances cold-induced browning of subcutaneous adipose tissue via promoting M2 macrophage proliferation," Cell Metabolism, vol. 22, no. 2, pp. 279-290, 2015.

[29] M. Margaritis, A. S. Antonopoulos, J. Digby et al., "Interactions between vascular wall and perivascular adipose tissue reveal novel roles for adiponectin in the regulation of endothelial nitric oxide synthase function in human vessels," Circulation, vol. 127, no. 22, pp. 2209-2221, 2013.

[30] S. Kajimura, B. M. Spiegelman, and P. Seale, "Brown and beige fat: physiological roles beyond heat generation," Cell Metabolism, vol. 22, no. 4, pp. 546-559, 2015.

[31] D. K. Wijesundara, D. C. Tscharke, R. J. Jackson, and C. Ranasinghe, "Reduced interleukin-4 receptor $\alpha$ expression on $\mathrm{CD}^{+} \mathrm{T}$ cells correlates with higher quality anti-viral immunity," PLoS One, vol. 8, no. 1, article e55788, 2013.

[32] T. Wang and C. J. Secombes, "The evolution of IL-4 and IL-13 and their receptor subunits," Cytokine, vol. 75, no. 1, pp. 8-13, 2015. 

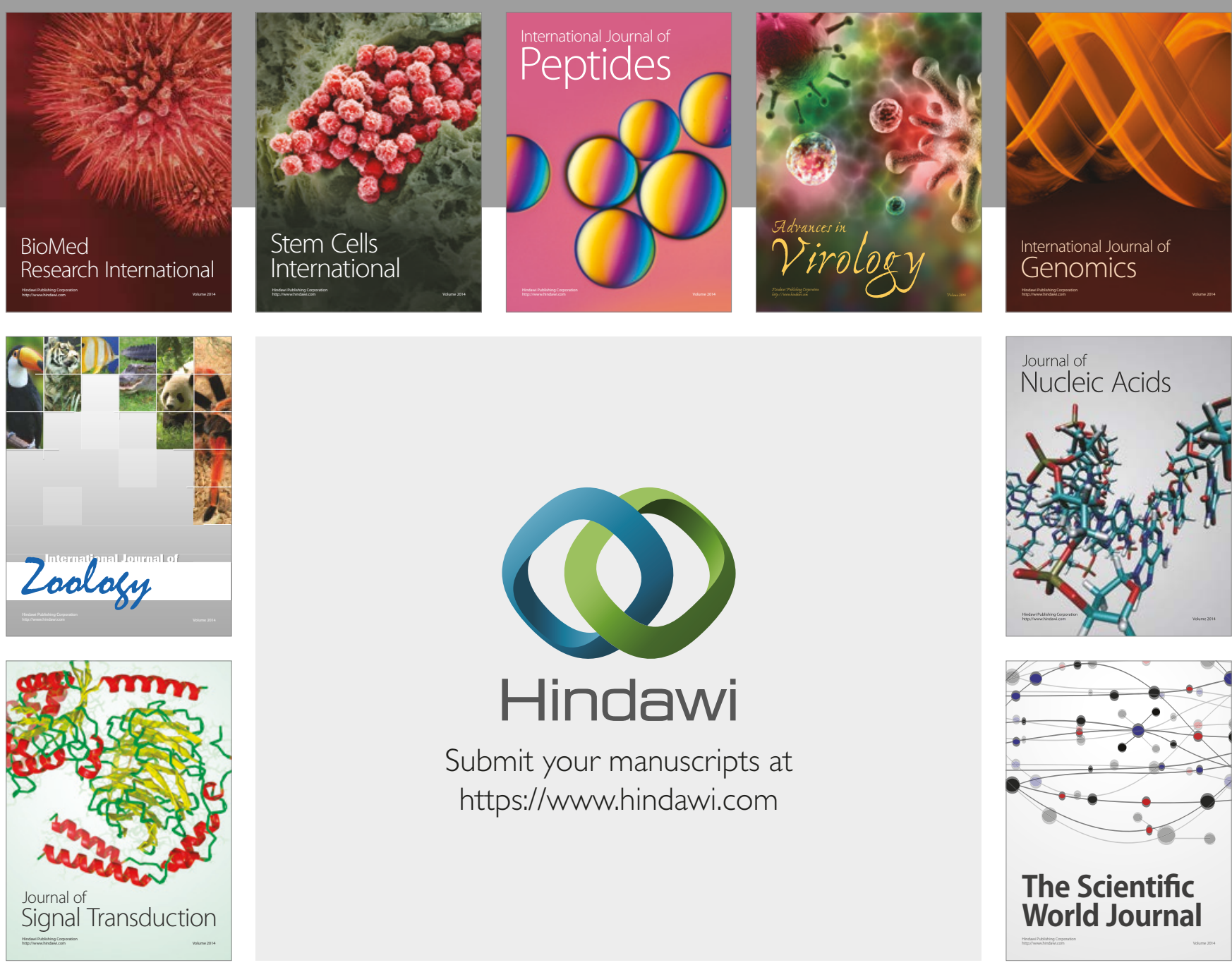

Submit your manuscripts at

https://www.hindawi.com
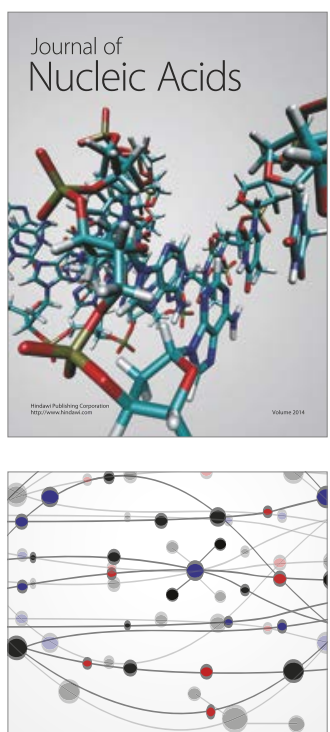

The Scientific World Journal

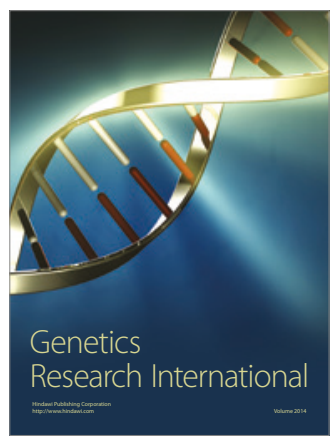

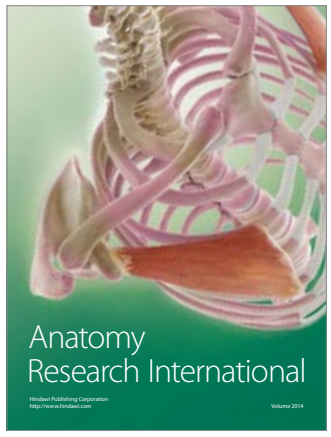

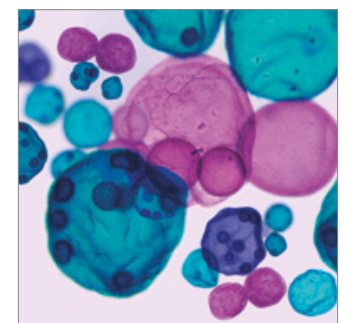

International Journal of Microbiology
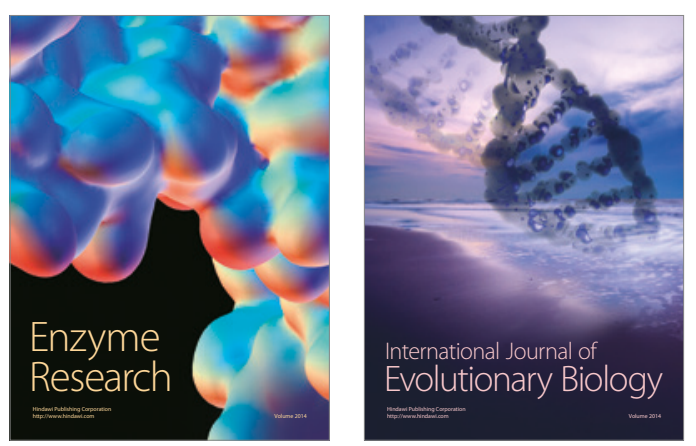
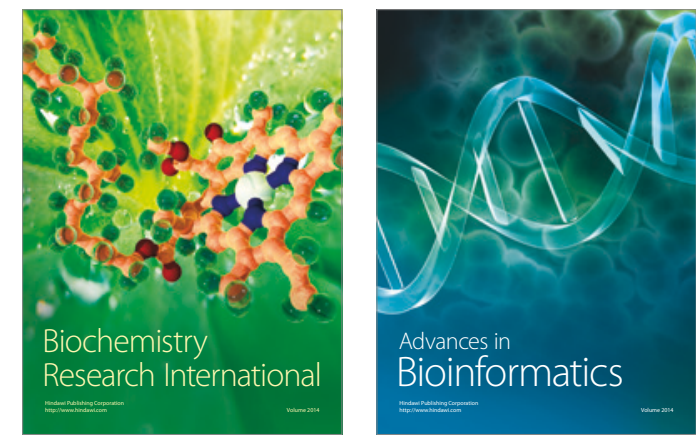

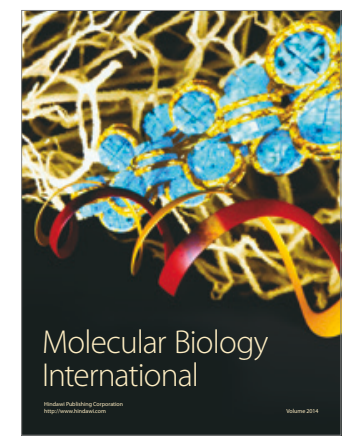

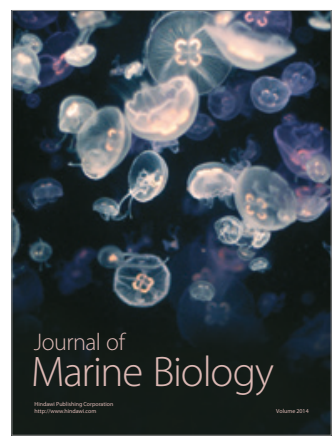

